\title{
Total bacterial counts on oral mucosa after using a commercial saliva substitute in patients undergoing hematopoietic cell transplantation
}

Yuko Sugiura ${ }^{1}$, Yoshihiko Soga ${ }^{1}$, Kokoro Yamabe ${ }^{1}$, Soichiro Tsutani ${ }^{1}$, Ichiro Tanimoto ${ }^{1}$, Hiroshi Maeda ${ }^{1}$, Susumu Kokeguchi ${ }^{2}$, Nobuharu Fujii ${ }^{3}$, Fumihiko Ishimaru ${ }^{3 *}$, Mitsune Tanimoto ${ }^{3}$, Fusanori Nishimura $^{1 * *}$, Shogo Takashiba ${ }^{1}$

1. Department of Pathophysiology - Periodontal Science, Okayama University Graduate School of Medicine, Dentistry and Pharmaceutical Sciences, Okayama, Japan

**Current address: Department of Dental Science for Health Promotion, Division of Cervico-Gnathostomatology, Hiroshima University Graduate School of Biomedical Sciences, Hiroshima, Japan

2. Department of Global Health and Environmental Sciences - Oral Microbiology, Okayama University Graduate School of Medicine, Dentistry and Pharmaceutical Sciences, Okayama, Japan

3. Department of Hematology, Oncology and Respiratory Medicine, Okayama University Graduate School of Medicine, Dentistry and Pharmaceutical Sciences, Okayama, Japan

*Current address: Okayama Red Cross Blood Center, Okayama, Japan

Corresponding author:

Shogo Takashiba, D.D.S., Ph.D.

Professor and Chair

e-mail: stakashi@cc.okayama-u.ac.jp

Department of Pathophysiology - Periodontal Science

Okayama University Graduate School of Medicine, Dentistry and Pharmaceutical Sciences

2-5-1 Shikata-cho, Okayama 700-8525, Japan

Tel: 81-86-235-6677

Fax: 81-86-235-6679

On behalf of Prof. S. Takashiba,

Yoshihiko Soga, D.D.S., Ph.D.

Assistant Professor

e-mail: y_soga@md.okayama-u.ac.jp 


\begin{abstract}
Purpose: The commercial saliva substitute Oralbalance ${ }^{\circledR}$ has been reported to alleviate symptoms of post-radiotherapy xerostomia in head and neck cancer patients. Oralbalance ${ }^{\circledR}$ may also be effective for xerostomia in patients undergoing hematopoietic cell transplantation (HCT) with high-dose chemotherapy and total-body irradiation. However, HCT patients are in a severely compromised condition, and saliva substitute must not promote infection. We reported previously that Oralbalance ${ }^{\circledR}$ has anti-microbial effects against microbial species detected during HCT in vitro. This study was performed to determine the in vivo effects of Oralbalance ${ }^{\circledR}$ on oral mucosal total bacterial counts in patients undergoing HCT.
\end{abstract}

Methods: A total of 18 neutropenic patients undergoing HCT were enrolled in this study. Before and after 1 week of Oralbalance ${ }^{\circledR}$ use, bacterial samples were obtained from patients by wiping an area of $\phi 1 \mathrm{~cm}$ on the buccal mucosa with sterilized cotton swabs. Total bacterial counts of the obtained samples were examined by quantitative polymerase chain reaction amplification of the bacterial $16 \mathrm{~S}$ ribosomal RNA gene. As controls, bacterial samples were also obtained from 10 healthy subjects, and total bacterial counts were examined.

Results: No significant increase in bacterial count was observed with use of Oralbalance ${ }^{\circledR}$. None of the patients showed bacterial counts above the range found in healthy controls after using Oralbalance ${ }^{\circledR}$.

Conclusions: In neutropenic patients undergoing HCT, Oralbalance ${ }^{\circledR}$ did not increase the total counts of oral mucosal bacteria beyond the range found in healthy controls. Oral care using Oralbalance ${ }^{\circledR}$ may alleviate the symptoms induced by hyposalivation without promoting infection.

Key Words: hematopoietic cell transplantation, xerostomia, saliva substitute 


\section{Introduction}

High-dose chemotherapy and total-body irradiation, which are performed as the conditioning regimen for hematopoietic cell transplantation (HCT), are associated with xerostomia. Oralbalance ${ }^{\circledR}$ (Laclede, Inc., Rancho Dominguez, CA, USA), which is a commercially available saliva substitute, has been reported to alleviate the symptoms of post-radiotherapy xerostomia in head and neck cancer patients [4,8]. Therefore, this product may be effective in HCT patients. However, as these patients are in a markedly compromised condition throughout the period of HCT, saliva substitute must not promote infection.

Previously, we reported the in vitro anti-microbial effects of Oralbalance ${ }^{\circledR}$ against microbial species detected during HCT [6]. Oralbalance ${ }^{\circledR}$ does not facilitate increases in microorganisms detected in the HCT period in vitro [6]. In the present study, we determine the in vivo effects of Oralbalance ${ }^{\circledR}$ on total bacterial counts of oral mucosa in neutropenic patients undergoing HCT.

\section{Subjects and Methods}

Subjects

A total of 18 neutropenic patients (neutrophil counts $<1,000 / \mu \mathrm{L}$ ) undergoing allogeneic conventional (not reduced intensity regimen) HCT with a conditioning regimen composed of total body irradiation (TBI) and high-dose chemotherapy at Okayama University Hospital (M, 12; F, 6; age, $42.9 \pm$ $16.2 \mathrm{y}$ ), who elected to use Oralbalance ${ }^{\circledR}$ to alleviate their symptoms of xerostomia, were enrolled in this 
study. The diseases in these patients were as follows: malignant lymphoma, 9; acute myeloid leukemia, 4; acute lymphocytic leukemia, 3; myelodysplastic syndromes, 1; solid tumor, 1. Ten of the 18 subjects did not require antibiotics, while the remaining 8 subjects needed antibiotics on at least one day during the examination period. Informed consent was obtained from all subjects, and the Ethics Committee of Okayama University Graduate School of Medicine, Dentistry and Pharmaceutical Sciences approved this study.

Oral managements and use of Oralbalance ${ }^{\circledR}$

All subjects were referred to dentists, and necessary dental treatment had been completed before HCT. All subjects were taught about the self management of oral hygiene; tooth brushing after every meal and before going to bed, and oral rinsing with normal saline solution every $3 \mathrm{~h}$ during the day was also indicated. Nurses, dental hygienists, and dentists performed this oral management in patients with poor general condition. They used Oralbalance ${ }^{\circledR}$ from the day on which the patients felt xerostomia (all patients began use of Oralbalance ${ }^{\circledR}$ from 5 to 1 days prior to HCT, which corresponded to the period of the conditioning regimen for HCT composed of TBI and high-dose chemotherapy) at least 4 times per day; i.e., after every meal and before going to bed.

Total counts of bacteria on the buccal mucosa

Bacterial samples were obtained about 2 hours after breakfast by wiping the buccal mucosa with sterilized cotton swabs over an area of $\phi 1 \mathrm{~cm}$ before and after 1 week of using Oralbalance ${ }^{\circledR}$. Samples were obtained from areas without ulcer. As controls, samples were also obtained from 10 healthy members 
of the hospital staff (M, 5; F, 5; age, $30.5 \pm 4.2$ y). Controls did not use Oralbalance ${ }^{\circledR}$ and were only sampled once. Total bacterial counts were examined by quantitative polymerase chain reaction (PCR) amplification of the bacterial 16S ribosomal RNA gene (16S rDNA) as described previously [3] with minor modifications as follows. Cotton swab samples were suspended in $1 \mathrm{~mL}$ of PBS(-) (Gibco BRL, Grand Island, NY, USA). Aliquots of $500 \mu \mathrm{L}$ from each suspension were transferred into new tubes and pelleted. Pelleted samples were resuspended in $200 \mu \mathrm{L}$ of InstaGene matrix (Bio-Rad Laboratories, Hercules, CA, USA) to extract total bacterial DNA. Aliquots of $5 \mu \mathrm{L}$ of extracted DNA were quantified by real-time PCR amplification of the 16S rDNA with SYBR Green. Real-time PCR and data analysis were performed using a GeneAmp ${ }^{\circledR} 5700$ Sequence Detection System and GeneAmp 5700 SDS software (Applied Biosystems, Foster City, CA, USA).

Statistical analysis

Total bacterial counts before and after the use of Oralbalance ${ }^{\circledR}$ were compared by Wilcoxon’s signed-rank test. $P$-values were calculated using the statistical software StatFlex (Artech, Osaka, Japan).

\section{Results}

Changes in total bacterial counts on the buccal mucosa after using Oralbalance ${ }^{\circledR}$

As shown in Fig. 1, there were no significant changes in total counts of bacteria on the buccal mucosa after use of Oralbalance ${ }^{\circledR}$. The group without antibiotic use (Fig. 1B) tended to have higher total bacterial counts than the group treated with antibiotics (Fig. 1C), although the difference was not 
significant. After using Oralbalance ${ }^{\circledR}$, none of the patients showed bacterial counts above the range found in healthy controls $\left(10^{4.2}-10^{5.6}, n=10\right)$.

\section{Discussion}

Our previous study demonstrated antimicrobial activity of Oralbalance ${ }^{\circledR}$ against the bacterial species detected during HCT in vitro, and suggested that this product would not promote infection. In the present study, we performed further examination of the in vivo effects of Oralbalance ${ }^{\circledR}$ on total bacterial counts of the oral mucosa in patients undergoing HCT. No significant increases in bacterial counts were observed associated with use of Oralbalance ${ }^{\circledR}$. It would be better to examine the differences with and without use of Oralbalance ${ }^{\circledR}$ as a case-control study. However, when this study was performed, the rumor that oral management with Oralbalance ${ }^{\circledR}$ alleviated oral pain spread among the patients in the ward. As a result, almost all patients requested oral management with Oralbalance ${ }^{\circledR}$, and a case-control study could not be performed. Therefore, we compared total bacterial counts on the oral mucosa of HCT patients with those of healthy subjects. None of the patients showed bacterial counts above the range found in healthy controls after using Oralbalance ${ }^{\circledR}$.

In this study, quantitative PCR amplification of 16S rDNA was performed to evaluate the total counts of bacteria on the oral mucosa. Samples were obtained from areas without ulcer because of pain, and oral ulceration may influence colonization by microorganisms. Oral care was performed intensively, and no visible colonization was observed in any of the subjects during the examination period. The gene 
encoding the small subunit of bacterial 16S rDNA has been used frequently as a target of PCR examination because of its structural characteristics [2,5]. The nucleotide sequences of some portions of the 16S rDNA are highly conserved through evolution [3]. The conserved sequences can provide PCR primers for amplification of 16S rDNA from all bacterial species [3]. Quantitative real-time PCR has been demonstrated to be a powerful tool for quantitative microbiological examination [3]. Therefore, we evaluated the changes in total bacterial counts on the buccal mucosa after using Oralbalance ${ }^{\circledR}$ by real-time PCR quantification of 16S rDNA. Culture and colony counting are common methods of determining bacterial counts. However, it can be difficult to culture some species of bacteria. Our results based on the bacterial DNA counts were reliable compared with culture-based methods.

The results of this study confirmed that Oralbalance ${ }^{\circledR}$ does not promote infection not only in vitro but also in vivo. Hyposalivation results in uncomfortable oral dryness, and may also increase the severity of the oral mucositis induced by chemotherapy and/or irradiation, because patients with xerostomia lose one of the most important factors involved in protecting the oral mucosa, saliva, which contains many components of the innate and acquired defense systems, and not only eliminates microorganisms from the oral cavity [1,7] but also moderates mechanical contact between the teeth and the oral mucosa. Indeed, we often see the development of ulcerative mucositis on the mucosa in contact with dry teeth clinically. Oral care using saliva substitute may alleviate the symptoms induced by hyposalivation without promoting infection. Furthermore, as Oralbalance ${ }^{\circledR}$ does not contain antibiotics, it does not contribute to the appearance of antibiotic-resistant bacteria. 
In conclusion, in neutropenic patients undergoing HCT, the commercially available saliva substitute Oralbalance ${ }^{\circledR}$ did not increase the total counts of oral mucosal bacteria beyond the range found in healthy controls.

\section{Acknowledgment}

This study was partly supported by a Grant-in-Aid for Young Scientists (Start-up) (20890138), Grant-in-Aid for Encouragement of Scientists (21933014) from the Japan Society for the Promotion of Science and the Scholarship Fund donated by the T\&K Corporation, Tokyo, Japan. The T\&K Corporation is the exclusive agent of Biotene ${ }^{\circledR}$ products including Oralbalance ${ }^{\circledR}$ (Laclede, Inc., Rancho Dominguez, CA, USA) in Japan. Okayama University Graduate School of Medicine, Dentistry and Pharmaceutical Sciences approved this private scholarship fund.

\section{References}

1. Brandtzaeg P (1989) Salivary immunoglobulins. In: Tenovuo J (ed) Human saliva: clinical chemistry and microbiology, Vol. II. CRC Press, Inc.: Boca Raton, FL, pp 1-54.

2. Harper-Owen R, Dymock D, Booth V, Weightman AJ, Wade WG (1999) Detection of unculturable bacteria in periodontal health and disease by PCR. J Clin Microbiol 37:1469-1473.

3. Maeda H, Fujimoto C, Haruki Y, Maeda T, Kokeguchi S, Petelin M, Arai H, Tanimoto I, Nishimura F, Takashiba S (2003) Quantitative real-time PCR using TaqMan and SYBR Green for 
Actinobacillus actinomycetemcomitans, Porphyromonas gingivalis, Prevotella intermedia, tetQ gene and total bacteria. FEMS Immunol Med Microbiol 39:81-86.

4. Shahdad SA, Taylor C, Barclay SC, Steen IN, Preshaw PM (2005) A double-blind, crossover study of Biotene Oralbalance and BioXtra systems as salivary substitutes in patients with post-radiotherapy xerostomia. Eur J Cancer Care (Engl) 14:319-326.

5. Slots J, Ashimoto A, Flynn MJ, Li G, Chen C (1995) Detection of putative periodontal pathogens in subgingival specimens by $16 \mathrm{~S}$ ribosomal DNA amplification with the polymerase chain reaction. Clin Infect Dis 20: 304-307.

6. Sugiura Y, Soga Y, Tanimoto I, Kokeguchi S, Nishide S, Kono K, Takahashi K, Fujii N, Ishimaru F, Tanimoto M, Yamabe K, Tsutani S, Nishimura F, Takashiba S (2008) Antimicrobial effects of the saliva substitute, Oralbalance ${ }^{\circledR}$, against microorganisms from oral mucosa in the hematopoietic cell transplantation period. Support Care Cancer 16:421-424.

7. Tenovuo J (1998) Antimicrobial function of human saliva - how important is it for oral health? Acta Odontol Scand 56:250-256.

8. Warde P, Kroll B, O'Sullivan B, Aslanidis J, Tew-George E, Waldron J, Maxymiw W, Liu FF, Payne D, Cummings B (2000) A phase II study of Biotene in the treatment of postradiation xerostomia in patients with head and neck cancer. Support Care Cancer 8:203-208. 


\section{Figure legend}

Fig. 1

Changes in total bacterial counts on the buccal mucosa with use of the mouth moisturizing gel,

Oralbalance ${ }^{\circledR}(n=18)$. All patients began use of Oralbalance ${ }^{\circledR}$ from 5 to 1 days prior to HCT. "Before" using Oralbalance ${ }^{\circledR}$ samples were obtained at these points. “After” samples were obtained 1 week after starting use of Oralbalance ${ }^{\circledR}$. A: All subjects $(n=18)$. B: No antibiotics $(n=10)$. C: Subjects used antibiotics at least once $(n=8)$. No significant increases in bacterial counts were observed associated with use of Oralbalance ${ }^{\circledR}$ (Wilcoxon's signed-rank test; ND: no significant difference, $P>0.05$ ). Bacterial counts of healthy controls are shown in the gray area $\left(10^{4.2}-10^{5.6}, n=10\right)$. None of the patients' counts were above the range of those in healthy controls. 
(A)

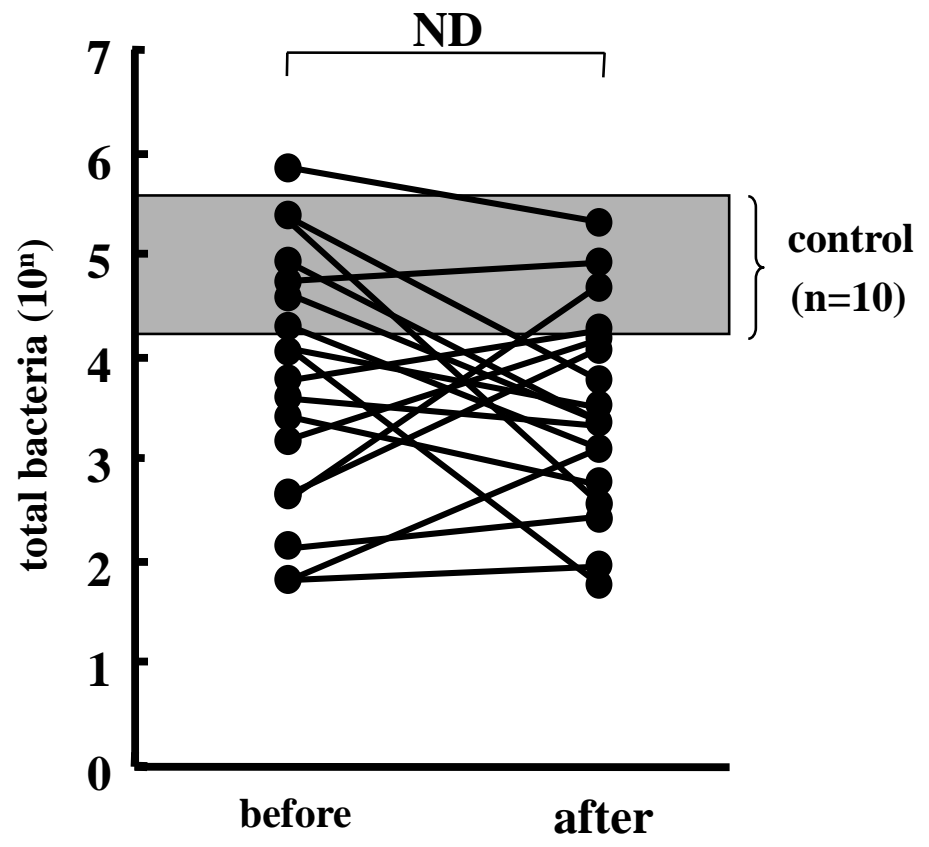

(B)

(C)
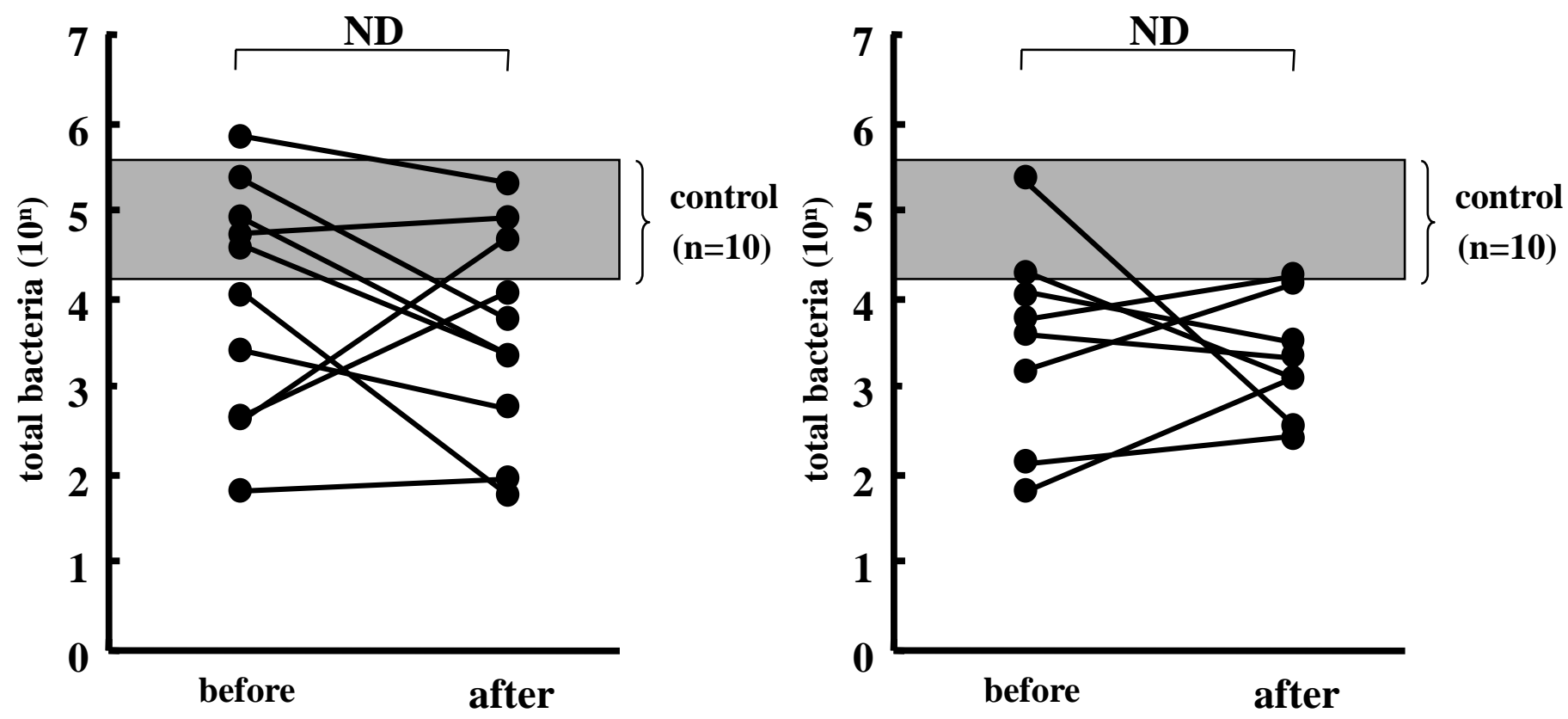